\title{
Comparison of sleep quality among puerperal women before and during the COVID-19 pandemic: a cross-sectional survey in Lanzhou, China
}

\author{
Shengping $\mathrm{Li}^{1,2,3} \cdot$ Lianlian Wang ${ }^{4}$. Wenling Wang ${ }^{5}$. Shengzhe Hou ${ }^{6}$. Changxiao Xie ${ }^{1,2,3} \cdot$ Mao Zeng ${ }^{1,2,3}$. \\ Jinli Xian ${ }^{1,2,3} \cdot$ Zhengjie Cai $^{1,2,3} \cdot$ Yong Zhao ${ }^{1,2,3,7}$
}

Received: 31 August 2021 / Revised: 10 November 2021 / Accepted: 15 December 2021 / Published online: 21 January 2022

(c) The Author(s), under exclusive licence to Springer Nature Switzerland AG 2021

\begin{abstract}
Purpose Few studies have yet examined sleep quality among puerperal women during the coronavirus disease 2019 (COVID19) pandemic. This cross-sectional study aimed to compare the sleep quality of puerperal women before and during the COVID-19 pandemic in Lanzhou, China. The study exploreed the factors that affect sleep quality among puerperal women. Methods The study population comprised puerperal women who went to the obstetric department of the Gansu Provincial Maternity and Childcare Hospital on the 42nd day after childbirth. Sleep quality was measured using the Pittsburgh Sleep Quality Index (PSQI). Data were collected by doctors in the obstetric department before the COVID-19 pandemic (Oct.-Dec. 2019) and during the COVID-19 pandemic (Feb.-Apr. 2020) in China. Multiple linear regressions were used to examine the association between the sleep quality of puerperal women and COVID-19 and to identify factors that affect the total PSQI scores.

Results In 2019, 546 puerperal women were assessed compared with 655 who were assessed in 2020. Compared with before COVID-19, the total PSQI scores of puerperal women improved during the COVID-19 pandemic. In addition, the influencing factors of sleep quality among puerperal women were the pandemic itself, age, conception method, and postpartum depression (PPD).

Conclusions In contrast with other studies, there was no evidence for worsening sleep quality of puerperal women during the COVID-19 pandemic in Lanzhou, China. Puerperal women who underwent assisted pregnancy or developed PPD were more likely to experience poor sleep quality.
\end{abstract}

Keywords COVID-19 $\cdot$ Puerperal women $\cdot$ Sleep quality, Comparison

Shengping Li and Lianlian Wang have contributed equally to this work and share first authorship.

Yong Zhao

zhaoyong@cqmu.edu.cn

1 School of Public Health and Management, Chongqing Medical University, Yi xueyuan Road, Yu zhong District, Chongqing 400016, China

2 Research Center for Medicine and Social Development, Chongqing Medical University, Chongqing, China

3 The Innovation Center for Social Risk Governance in Health, Chongqing Medical University, Chongqing, China
4 The Department of Obstetrics, The First Affiliated Hospital of Chongqing Medical University, Chongqing, China

5 Perinatology Center, Gansu Provincial Maternity and Child-Care Hospital, Lanzhou, Gansu, China

6 The Second Clinical College, Chongqing Medical University, Chongqing, China

7 Chongqing Key Laboratory of Child Nutrition and Health, Children's Hospital of Chongqing Medical University, Chongqing, China 


\section{Introduction}

The coronavirus disease 2019 (COVID-19) pandemic has become a serious global crisis, profoundly influencing daily life and posing a major public health challenge. A recent review that included 50 studies found that the prevalence rate of psychological morbidity was $44 \%$ due to the COVID-19 pandemic, with poor sleep quality accounting for the highest proportion, followed by stress, anxiety, and depression [1].

Puerperal women are facing major life changes and challenges that involve drastic changes at the social, biological, and psychological levels [2]. Puerpera is a high-risk period for the development of mood disorders [3]. Puerperal women frequently experience difficulty in obtaining adequate sleep quality or quantity. Puerperal women normally have less sleep time and more time awake [4], particularly in the first postpartum month [5]. Poor sleep quality during the puerperal period is associated with symptoms of depression [6], anxiety [7], and a decrease in daytime attentiveness [8], affecting puerperal women, their babies, and their families [9]. By contrast, high-quality sleep is the basis for the physical recovery of puerperal women. It is also an important indicator of physical and mental health and quality of life.

Most previous studies have focused on the effects of COVID-19 on the mental health of medical healthcare workers and the general public; these effects include poor sleep quality, depression, anxiety, and psychological distress [10]. The COVID-19 pandemic may impair the sleep quality of pregnant women [11]. Two cross-sectional studies found that $88 \%$ of pregnant women suffered from poor sleep quality during the COVID-19 pandemic [12], including difficulty falling sleep, short sleep duration, and ordinary or poor subjective sleep quality [13].

Few studies have yet examined sleep quality among puerperal women during the COVID-19 pandemic. Sleep disorders are common among puerperal women. Thus, the current study aimed to (1) compare sleep quality among puerperal women before and during the COVID-19 pandemic and (2) identify the factors that affect sleep quality.

\section{Methods}

\section{Study design}

A cross-sectional survey was conducted among puerperal women who went to the Gansu Provincial Maternity and Childcare Hospital in Lanzhou, a city in Northwest China, on the 42nd day after childbirth. Data were collected from October to December in 2019 and February to April in 2020. Participants were puerperal women examined in the obstetrics department of the aforementioned hospital. Puerperal women who were 18-45 years old completed the questionnaire. A total of 1201 participants were included in the analysis. The first period of data collection was from October to December 2019. These participants belonged to the "before the COVID-19 pandemic" (T1) group. The second period of data collection was from February to April 2020. These participants belonged to the "during the COVID-19 pandemic" (T2) group. This study was approved by the Ethics Committee of the First Affiliated Hospital of Chongqing Medical University on October 22, 2018, and the approval number was 2018-131. All the participants provided informed consent.

\section{Data collection}

\section{Demographic, socioeconomic, and obstetric characteristics}

Data were collected by doctors in the obstetric clinic. Data regarding the participants' sociodemographic characteristics (e.g., age, educational level, residence, and per capital monthly family income), infant feeding pattern, delivery method, parity, and conception method were collected. Educational level was categorized into low (junior middle school or below), medium (senior high school, vocational, or technical secondary school), and high (university or college) [14]. Residence included urban or rural areas. Per capital monthly family income was grouped into low (<¥4500), medium ( $¥ 4500-¥ 9000$ ), and high ( $>¥ 9000$ ) [14]. 1 USD $=¥ 7.01$ in February 2020. Infant feeding pattern included exclusive breastfeeding and others (i.e., bottle feeding or mixed feeding). Delivery method was either vaginal delivery or cesarean section. Parity included primipara and multipara. Conception method was either natural pregnancy or assisted pregnancy. The Edinburgh Postnatal Depression Scale (EPDS) was used to screen for postpartum depression (PPD) [15], and an EPDS score of $>12$ was defined as PPD [16].

\section{Assessments of sleep quality}

The Pittsburgh Sleep Quality Index (PSQI) scale (Chinese version), which was translated and validated by Liu [17], was used to measure the sleep quality of puerperal women. PSQI is a self-rated questionnaire with 19 items designed to measure sleep quality and disturbance over the past month [18]. The PSQI are grouped into seven components: subjective sleep quality (C1), sleep latency (C2), sleep duration (C3), habitual sleep efficiency (C4), sleep disturbance (C5), use of sleeping medication (C6), and daytime dysfunction (C7). We added crying baby, changing soiled diapers, and feeding the baby to sleep disturbance (C5), yielding a score that ranges 
from 0 to 36 . Proportionally generating $0-3$ points for this part, 0 had a score of $0,1-12$ had a score of 1, 13-24 had a score of 2, and 25-36 had a score of 3. Each sleep component yields a score that ranges from 0 to 3 , with 3 indicating the greatest dysfunction. The sleep component scores are summed to yield the total PSQI score, which ranges from 0 to 21. A higher total score (referred to as the global score) indicates worse sleep quality. Liu reported that a total PSQI score of $>7$ exhibits good sensitivity and specificity when distinguishing between good and poor sleepers [17].

\section{Statistical analysis}

Descriptive statistics were used for the sample characteristics. The categorical variables were described using frequency and percentage, while the continuous variables were described using mean and standard deviation. The comparison of the participants' sociodemographic and obstetric characteristics between $\mathrm{T} 1$ and $\mathrm{T} 2$ was achieved via a chisquare test except age, which was normally distributed, and used the $t$-test. We used the Shapiro-Wilk test to analyze the normality of the sleep quality score, including the scores in PSQI and the seven components, which were found to be normally distributed. The analysis of the comparison between sleep quality scores before and during the COVID19 pandemic was achieved using an independent $t$-test. A multiple linear regression model was used to identify factors that affect the sleep quality of puerperal women. We adopted PSQI score as the dependent variable in the regression model. Meanwhile, survey time (T1 and T2), age, educational level, residence, per capital monthly family income, infant feeding pattern, delivery method, parity, conception method, and PPD were used as the independent variables in the regression model. All analyses were performed using STATA 16.1 (Stata Corporation, College Station). Statistical significance was considered when $p<0.05$ (two-sided).
Table 1 Characteristics of study participants $(n=1201)$

\begin{tabular}{|c|c|c|c|c|c|}
\hline Variables & $\begin{array}{l}\text { All par- } \\
\text { ticipants } \\
(n=1201)\end{array}$ & $\mathrm{T} 1(n=546)$ & $\mathrm{T} 2(n=655)$ & $t / \chi^{2}$ & $p$-value \\
\hline Age (years) (mean (SD)) & $29.97(3.6)$ & $29.36(3.5)$ & $30.49(3.7)$ & 5.43 & $<0.001 * * *$ \\
\hline Education & & & & 5.44 & 0.07 \\
\hline Low & $109(9)$ & $41(7)$ & $68(10)$ & & \\
\hline Medium & $77(6)$ & $29(5)$ & $48(7)$ & & \\
\hline High & $1015(84)$ & $476(87)$ & $539(82)$ & & \\
\hline Residence & & & & 0.39 & 0.53 \\
\hline Urban & $1119(93)$ & $506(93)$ & $613(93)$ & & \\
\hline Rural & $82(7)$ & $40(7)$ & $42(6)$ & & \\
\hline Per capital monthly family income & & & & 6.05 & 0.05 \\
\hline Low & $289(24)$ & $114(21)$ & $175(27)$ & & \\
\hline Medium & $444(37)$ & $205(38)$ & $239(36)$ & & \\
\hline High & $468(39)$ & $227(42)$ & $241(37)$ & & \\
\hline Exclusive breastfeeding & & & & 20.22 & $<0.001 * * *$ \\
\hline Yes & $623(52)$ & $322(59)$ & $301(46)$ & & \\
\hline No & $578(48)$ & $224(41)$ & $354(54)$ & & \\
\hline Delivery way & & & & 8.51 & $<0.001 * * *$ \\
\hline Cesarean section & $402(34)$ & $159(29)$ & $243(37)$ & & \\
\hline Vaginal delivery & $799(66)$ & $387(71)$ & $412(63)$ & & \\
\hline Parity & & & & 1.54 & 0.22 \\
\hline Primipara & $834(69)$ & $389(71)$ & $445(68)$ & & \\
\hline Multipara & $367(31)$ & $157(29)$ & $210(32)$ & & \\
\hline Conception method & & & & 2.75 & 0.09 \\
\hline Natural pregnancy & $1124(94)$ & $518(95)$ & $606(92)$ & & \\
\hline Assisted pregnancy & $77(6)$ & $28(5)$ & $49(8)$ & & \\
\hline Postpartum depression & & & & 2.50 & 0.11 \\
\hline No & $895(75)$ & $395(72)$ & $500(76)$ & & \\
\hline Yes & $306(25)$ & $151(28)$ & $155(24)$ & & \\
\hline
\end{tabular}

Chi-square test, independent $t$-test. $* P<0.05, * * P<0.01$, *** $P<0.001$

$T 1$, before the COVID-19 pandemic; T2, during the COVID-19 pandemic 


\section{Results}

The demographic, socioeconomic, and obstetric characteristics of the participants are provided in Table 1. Among the 1201 participants, the mean age was $30.0 \pm 3.6$ years. The majority of the participants achieved high education $(84 \%, n=1015)$, lived in an urban area $(93 \%, n=1119)$, and got pregnant through natural conception $(94 \%, n=1124)$. Compared with that of the participants in T1 $(n=546)$, the average age of the participants in T2 $(n=655)$ was slightly higher $(29.4 \pm 3.5$ versus $30.5 \pm 3.7)$. Furthermore, the number of participants who chose exclusive breastfeeding decreased, and more participants gave birth through cesarean section.

The comparison of the scores in PSQI and the seven components of sleep quality of puerperal women before and during the COVID-19 pandemic are presented in Table 2. The total PSQI scores of $\mathrm{T} 1$ were statistically higher than that of $\mathrm{T} 2(7.9 \pm 3.1$ versus $7.3 \pm 3.1$ ). The mean scores of subjective sleep quality, sleep duration, habitual sleep efficiency, sleep disturbance, and daytime dysfunction before the COVID-19 pandemic were also statistically higher than those during the COVID-19 pandemic $(1.3 \pm 0.7$ versus $1.2 \pm 0.7,1.2 \pm 0.9$ versus $1.1 \pm 0.9,1.8 \pm 1.2$ versus $1.6 \pm 1.2,1.2 \pm 0.5$ versus $1.1 \pm 0.5$, and $1.7 \pm 1.0$ versus $1.5 \pm 0.9$, respectively). All $p$ values were $<0.05$.

Four items reflected habitual sleep efficiency. Compared with those for the participants in $\mathrm{T} 1$, the times to sleep at night and wake up in the morning were later for the participants in $\mathrm{T} 2(22.5 \pm 1.2$ versus $23.0 \pm 1.1$ and $7.5 \pm 1.0$ versus $8.0 \pm 1.1$ ). The sleep time per night of the participants in $\mathrm{T} 2$ was longer, and their sleep efficiency was higher $(6.2 \pm 1.4$ versus $6.4 \pm 1.5$ and $70 \%$ versus $72 \%)$. All $p$ values were $<0.05$. Eleven items reflected sleep disturbance. Compared with the puerperal women in T1, the puerperal women in $\mathrm{T} 2$ had significantly lower mean scores in crying baby, changing soiled diapers, and feeding the baby.

Table 3 lists the factors that affect the total PSQI scores of puerperal women. We used the total PSQI score as the dependent variable in the regression model. Meanwhile, survey time (before the COVID-19 pandemic and during the COVID-19 pandemic), age, educational level, residence, per capital monthly family income, infant feeding pattern, delivery method, parity, conception method, and postpartum depression were adopted as the independent variables in the regression model. In accordance with the results of the multiple linear regressions, the factors included survey
Table 2 Comparison of sleep quality of participants between before and during the COVID19 pandemic $(n=1201)$

\begin{tabular}{lllc}
\hline Variables & T1 $(n=546)$ & T2 $(n=655)$ & $p$-value \\
\hline The total PSQI scores (mean (SD)) & $7.9(3.1)$ & $7.3(3.1)$ & $<0.001^{* * *}$ \\
Seven components of sleep quality & & & \\
C1. Subjective sleep quality (mean (SD)) & $1.3(0.7)$ & $1.2(0.7)$ & $<0.001^{* * *}$ \\
C2. Sleep latency (mean (SD)) & $0.7(0.7)$ & $0.7(0.8)$ & 0.73 \\
C3. Sleep duration (mean (SD)) & $1.2(0.9)$ & $1.1(0.9)$ & $0.02^{*}$ \\
C4. Habitual sleep efficiency (mean (SD)) & $1.8(1.2)$ & $1.6(1.2)$ & $0.02^{*}$ \\
Time to sleep at night (h) & $22.5(1.2)$ & $23.0(1.1)$ & $0.04 *$ \\
Time to wake up in the morning (h) & $7.5(1.0)$ & $8.03(1.1)$ & $<0.01^{* *}$ \\
Sleep time per night (h) & $6.2(1.4)$ & $6.4(1.5)$ & $<0.001 * * *$ \\
Sleep efficiency (\%) & $70.0(16)$ & $72.4(16)$ & $<0.01 * *$ \\
C5. Sleep disturbance (mean (SD)) & $1.2(0.5)$ & $1.1(0.5)$ & $0.04 *$ \\
Wake up in the middle night or early morning & $0.6(1.0)$ & $0.55(1.0)$ & 0.63 \\
Have to get up to use the bathroom & $1.0(1.1)$ & $0.9(1.1)$ & 0.11 \\
Cannot breathe comfortably & $0.1(0.3)$ & $0.1(0.3)$ & 0.85 \\
Cough or snore loudly & $0.1(0.5)$ & $0.2(0.6)$ & 0.45 \\
Feel too cold & $0.2(0.7)$ & $0.3(0.7)$ & 0.23 \\
Feel too hot & $0.7(1.1)$ & $0.6(1.1)$ & 0.35 \\
Had bad dreams & $0.3(0.7)$ & $0.4(0.8)$ & 0.14 \\
Have pain & $0.3(0.7)$ & $0.3(0.7)$ & 0.56 \\
Baby crying & $1.6(1.2)$ & $1.4(1.2)$ & $0.03^{*}$ \\
Change wet diapers & $2.0(1.2)$ & $1.68(1.3)$ & $<0.001^{* * *}$ \\
Feeding baby & $2.3(1.1)$ & $2.0(1.2)$ & $<0.001^{* * *}$ \\
C6. Use of sleeping medication (mean (SD)) & $0.02(0.2)$ & $0.01(0.1)$ & 0.771 \\
C7. Daytime dysfunction (mean (SD)) & $1.7(1.0)$ & $1.5(0.9)$ & $<0.001 * * *$ \\
\hline
\end{tabular}

Independent $t$-test. $* P<0.05, * * P<0.01, * * * P<0.001$

$T 1$, before the COVID-19 pandemic; T2, during the COVID-19 pandemic 
Table 3 Multiple linear regressions to identify factors that affect the total PSQI scores $(n=1201)$

\begin{tabular}{|c|c|c|c|c|c|}
\hline Variables & $\beta$ & SE & $95 \% \mathrm{CI}$ & $t$ & $p$-value \\
\hline \multicolumn{6}{|l|}{ Survey time } \\
\hline \multicolumn{6}{|c|}{ Before the COVID-19 pandemic (Ref) } \\
\hline During the COVID-19 pandemic & -0.071 & 0.18 & $-0.80 \sim-0.09$ & -2.47 & $<0.01 * *$ \\
\hline Age & -0.069 & 0.03 & $-0.11 \sim 0.00$ & -2.11 & $0.04 *$ \\
\hline \multicolumn{6}{|l|}{ Education } \\
\hline \multicolumn{6}{|l|}{ Low (Ref) } \\
\hline Medium & -0.001 & 0.45 & $-0.90 \sim 0.87$ & -0.03 & 0.98 \\
\hline High & 0.045 & 0.32 & $-0.24 \sim 1.02$ & 1.21 & 0.23 \\
\hline \multicolumn{6}{|l|}{ Residence } \\
\hline \multicolumn{6}{|l|}{ Urban (Ref) } \\
\hline Rural & -0.006 & 0.36 & $-0.78 \sim 0.63$ & -0.21 & 0.83 \\
\hline \multicolumn{6}{|l|}{ Per capital monthly family income } \\
\hline \multicolumn{6}{|l|}{ Low (Ref) } \\
\hline Medium & 0.068 & 0.23 & $-0.02 \sim 0.90$ & 1.88 & 0.06 \\
\hline High & 0.037 & 0.23 & $-0.22 \sim 0.70$ & 1.03 & 0.30 \\
\hline \multicolumn{6}{|l|}{ Exclusive breastfeeding } \\
\hline \multicolumn{6}{|l|}{ Yes (Ref) } \\
\hline No & -0.008 & 0.18 & $-0.40 \sim 0.30$ & -0.28 & 0.78 \\
\hline \multicolumn{6}{|l|}{ Delivery way } \\
\hline \multicolumn{6}{|l|}{ Cesarean section (Ref) } \\
\hline Vaginal delivery & 0.020 & 0.19 & $-0.24 \sim 0.51$ & 0.70 & 0.48 \\
\hline \multicolumn{6}{|l|}{ Parity } \\
\hline \multicolumn{6}{|l|}{ Primipara (Ref) } \\
\hline Multipara & 0.009 & 0.22 & $-0.36 \sim 0.49$ & 0.29 & 0.78 \\
\hline \multicolumn{6}{|l|}{ Conception method } \\
\hline \multicolumn{6}{|l|}{ Natural pregnancy (Ref) } \\
\hline Assisted pregnancy & 0.064 & 0.36 & $0.11 \sim 1.53$ & 2.26 & $0.02 *$ \\
\hline \multicolumn{6}{|l|}{ Postpartum depression } \\
\hline \multicolumn{6}{|l|}{ No (Ref) } \\
\hline Yes & 0.23 & 0.20 & $1.26 \sim 2.05$ & 8.18 & $<0.001 * * *$ \\
\hline
\end{tabular}

time, age, conception method, and postpartum depression. When controlling for the participants' other sociodemographic and obstetric characteristics, compared with before the COVID-19 pandemic, puerperal women who were during the COVID-19 pandemic had higher sleep quality $(\beta=-0.071, p<0.01)$, and their sleep quality was positively correlated with their age $(\beta=-0.069, p=0.04)$. Compared with natural conception, puerperal women who underwent assisted pregnancy had worse sleep quality $(\beta=0.064$, $p=0.02)$. Postpartum depression was also a risk factor in the sleep quality of puerperal women $(\beta=0.230, p<0.001)$.

\section{Discussion}

This study primarily contributes to the limited literature on the comparison between the sleep quality of puerperal women before and during the COVID-19 pandemic. We found no evidence for worsening sleep quality of puerperal women during the COVID-19 pandemic in Lanzhou, China. The results of the multiple linear regressions showed that the sleep quality of puerperal women was affected by the COVID-19 pandemic itself, age, conception method, and postpartum depression.

In contrast with the studies conducted on the general public, health workers [19], and pregnant women [13], the puerperal women in the current study had better sleep quality during the COVID-19 pandemic than before the COVID-19 pandemic. This finding is worth exploring. First, during the home quarantine period, the time during which puerperal women lived together with family members increased significantly. Thus, they received more support from their family and their burden, such as changing soiled diapers and feeding the baby, was reduced. The support from their partners, families, and friends was associated with the increase in sleep duration [20] and the reduced anxiety [21]. Second, the Chinese government quickly implemented a policy of home 
quarantine and mobilized considerable resources to ensure that every family had sufficient food and medicines. This policy significantly reduced the risk of getting 2019-nCoV infection by going out to buy food or medicines. Moreover, people did not have to worry about food or medicine shortage during the home quarantine period. Lastly, working from home ensured a steady family income even during the COVID-19 pandemic, this may be associated with the remission of sleep disorders [22].

In the present study, we found no evidence for worsening sleep quality of puerperal women during the COVID19 pandemic in Lanzhou, China. The result was consistent with a study conducted in the UK [23]. Besides, a study in February 2020 of Hubei Province, which was considered to be the core of the outbreak of the COVID-19 pandemic [24], showed that sleep quality was significantly improved in people aged between $25-39$ years and $>60$ years, and most people in Hubei Province developed a more positive attitude regarding their risk of infection and the chances of surviving the COVID-19 epidemic [25]. It may be explained as follows. There began to be a gradual return to work with increasing numbers of people recovering from infection, and the accuracy of official public information improved, likely reducing the fear of the unknown [26], all of which gave the population some confidence.

Interestingly, the current study found that the sleeping and waking times of puerperal women became later during the COVID-19 pandemic compared with those before the COVID-19 pandemic. A study conducted in Italy also proved this result [27]. In addition, puerperal women had a longer sleep time per night and higher sleep efficiency. These results are consistent with those of a study conducted in the UK [23]. However, the proportion of good sleepers decreased, which is contrary to the results of our research. This difference can be explained by several possible reasons. First, different studies have used varying methods to evaluate the sleep quality of the study subjects, although the PSQI scale has been widely used. Second, we used a total PSQI score of $>7$ to distinguish between good and poor sleepers [17], which is inconsistent with other foreign studies that used a total PSQI score of $>5$ [18]. Notably, delayed sleep time may have long-term unfavorable effects, particularly for puerperal women with a high risk of PPD [28, 29].

Multiple linear regressions showed that age, conception method, and PPD affected the total PSQI scores of puerperal women in the current study. The participants' sleep quality was positively correlated with the age of the participants during the COVID-19 pandemic, and one study showed that older women are calmer and more relaxed than younger women [30]. Compared with natural pregnancy, puerperal women who underwent assisted pregnancy have worse sleep quality. Psychological distress, such as anxiety, stress, and guilt, during assisted reproductive therapy exerts a significant effect on sleep disorders [31, 32]. In accordance with a systematic review and a meta-analysis, the prevalence of PPD changed from 12 to $22 \%$ before and during the pandemic [33, 34]. In addition, an online survey in Belgium found that the prevalence of PPD was $23.6 \%$ [35]. This value is consistent with the results of our research $(24 \%)$. The aforementioned studies also found that PPD was a risk factor for sleep quality during the COVID-19 pandemic.

Our study has several strengths. First, this work is the first to focus on the sleep quality of puerperal women in China during the COVID-19 pandemic. Second, this study collected the sleep quality of puerperal women before the COVID-19 pandemic, and compared it with the sleep quality of puerperal women during the COVID-19 pandemic. Nevertheless, this study also has several limitations that should be acknowledged. First, this work used cross-sectional survey data and did not permit a reliable inference of causality. Longitudinal studies are necessary to examine the causal role of COVID-19 in sleep quality among puerperal women. Second, this study only included sociodemographic and obstetric characteristics in analyzing the factors that affect the sleep quality of puerperal women during the COVID-19 pandemic. More relevant factors, such as exercise [36] and eating habit [37], should be included in future studies. In addition, the measurement of sleep quality is self-reported using the PSQI scale. This evaluation index is subjective, and thus, an objective evaluation is lacking.

\section{Conclusions}

Puerperal women reported better sleep quality during the COVID-19 pandemic than before the pandemic in Lanzhou, China. However, puerperal women took longer to fall asleep at night, and risk of staying up late increased during the COVID-19 pandemic. In addition, puerperal women who underwent assisted pregnancy or developed PPD were more likely to experience poor sleep quality during the COVID-19 pandemic.

Acknowledgements We are grateful to the doctors of the obstetric department of the Gansu Provincial Maternity and Childcare Hospital for providing the data. The authors also wish to thank the study participants for their support in conducting this research.

Author contribution SL and SH contributed to the analysis and interpretation of data, drafted the report, and received the final version for publication. WW, CX, MZ, JX, ZC, LW, and YZ contributed to the analysis and interpretation of the data, commented on the report, revised the manuscript, and approved the final version for publication.

Funding This project was funded by Chongqing Social Science Planning Project (2017YBSH057) and Joint project of the Ministry of 
Technology and the Ministry of Health (2021MSXM215) and Discipline Cultivation Fund of the First Affiliated Hospital of Chongqing Medical University. The funders had no role in design, analysis, data interpretation and publication of the finding.

Data availability The data that support the findings of this study are available from the corresponding author upon request.

Code availability The code for analyzing the results of this study can be obtained from the corresponding author.

\section{Declarations}

Ethics approval and consent to participate The study was conducted in accordance with the Declaration of Helsinki, and the protocol was approved by the Ethics Committee of Chongqing Medical University. All the participants provided informed consent.

Conflict of interest The authors declare no competing interests.

\section{References}

1. Krishnamoorthy Y, Nagarajan R, Saya GK, Menon V (2020) Prevalence of psychological morbidities among general population, healthcare workers and COVID-19 patients amidst the COVID-19 pandemic: a systematic review and meta-analysis. Psychiatry Res 293:113382. https://doi.org/10.1016/j.psychres.2020.113382

2. Jeong YJ, Nho JH, Kim HY, Kim JY(2021) Factors influencing quality of life in early postpartum women. Int J Environ Res Public Health 18(6). https://doi.org/10.3390/ijerph18062988

3. Pearlstein T, Howard M, Salisbury A, Zlotnick C (2009) Postpartum depression. Am J Obstet Gynecol 200(4):357-364. https:// doi.org/10.1016/j.ajog.2008.11.033

4. Goyal D, Gay CL, Lee KA (2007) Patterns of sleep disruption and depressive symptoms in new mothers. J Perinat Neonatal Nurs 21(2):123-129. https://doi.org/10.1097/01.Jpn.0000270629. 58746.96

5. Lee KA, Zaffke ME, McEnany G (2000) Parity and sleep patterns during and after pregnancy. Obstet Gynecol 95(1):14-18. https:// doi.org/10.1016/s0029-7844(99)00486-x

6. Iranpour S, Kheirabadi GR, Esmaillzadeh A, Heidari-Beni M, Maracy MR (2016) Association between sleep quality and postpartum depression. J Res Med Sci 21:110. https://doi.org/10.4103/ 1735-1995.193500

7. Okun ML, Mancuso RA, Hobel CJ, Schetter CD, Coussons-Read M (2018) Poor sleep quality increases symptoms of depression and anxiety in postpartum women. J Behav Med 41(5):703-710. https://doi.org/10.1007/s10865-018-9950-7

8. Rychnovsky J, Hunter LP (2009) The relationship between sleep characteristics and fatigue in healthy postpartum women. Womens Health Issues 19(1):38-44. https://doi.org/10.1016/j.whi.2008.07. 015

9. Letourneau NL, Dennis CL, Benzies K et al (2012) Postpartum depression is a family affair: addressing the impact on mothers, fathers, and children. Issues Ment Health Nurs 33(7):445-457. https://doi.org/10.3109/01612840.2012.673054

10. Vindegaard N, Benros ME (2020) COVID-19 pandemic and mental health consequences: systematic review of the current evidence. Brain Behav Immun 89:531-542. https://doi.org/10. 1016/j.bbi.2020.05.048

11. Xie M, Wang X, Zhang J, Wang Y (2021) Alteration in the psychologic status and family environment of pregnant women before and during the COVID-19 pandemic. Int J Gynaecol Obstet 153(1):71-75. https://doi.org/10.1002/ijgo.13575

12. Alan S, Vurgec BA, Cevik A, Gozuyesil E, Surucu SG (2020) The effects of COVID-19 pandemic on pregnant women: perceived stress, social support and sleep quality. Yonago Acta Med 63(4):360-367. https://doi.org/10.33160/yam.2020.11.016

13. Lin W, Wu B, Chen B et al (2021) Sleep conditions associate with anxiety and depression symptoms among pregnant women during the epidemic of COVID-19 in Shenzhen. J Affect Disord 281:567-573. https://doi.org/10.1016/j.jad.2020.11.114

14. Xu X, Liu D, Zhang Z, Sharma M, Zhao Y (2017) Sleep duration and quality in pregnant women: a cross-sectional survey in China. Int J Environ Res Public Health 14(7). https://doi.org/ 10.3390/ijerph14070817

15. Cox JL, Holden JM, Sagovsky R (1987) Detection of postnatal depression. Development of the 10-item Edinburgh Postnatal Depression Scale. Br J Psychiatry 150:782-786. https://doi.org/ 10.1192/bjp.150.6.782

16. Levis B, Negeri Z, Sun Y, Benedetti A, Thombs BD (2020) Accuracy of the Edinburgh Postnatal Depression Scale (EPDS) for screening to detect major depression among pregnant and postpartum women: systematic review and meta-analysis of individual participant data. BMJ 371:m4022. https://doi.org/ 10.1136/bmj.m4022

17. Liu Xianchen TM, Hu Lei et al Reliability and validity of the Pittsburgh Sleep Quality Index. Chin J Psychiatry 1996:103107. In Chinese

18. Buysse DJ, Reynolds CF 3rd, Monk TH, Berman SR, Kupfer DJ (1989) The Pittsburgh Sleep Quality Index: a new instrument for psychiatric practice and research. Psychiatry Res 28(2):193213. https://doi.org/10.1016/0165-1781(89)90047-4

19. Wang S, Zhang Y, Ding W et al (2020) Psychological distress and sleep problems when people are under interpersonal isolation during an epidemic: a nationwide multicenter cross-sectional study. Eur Psychiatry 63(1):e77. https://doi.org/10.1192/j. eurpsy.2020.78

20. Faleschini S, Millar L, Rifas-Shiman SL, Skouteris H, Hivert MF, Oken E (2019) Women's perceived social support: associations with postpartum weight retention, health behaviors and depressive symptoms. BMC Womens Health 19(1):143. https:// doi.org/10.1186/s12905-019-0839-6

21. Liu H, Yang Y (2021) Effects of a psychological nursing intervention on prevention of anxiety and depression in the postpartum period: a randomized controlled trial. Ann Gen Psychiatry 20(1):2. https://doi.org/10.1186/s12991-020-00320-4

22. Wright L, Steptoe A, Fancourt D (2021) Are adversities and worries during the COVID-19 pandemic related to sleep quality? Longitudinal analyses of 46,000 UK adults. PLoS ONE 16(3):e0248919. https://doi.org/10.1371/journal.pone.0248919

23. Stanley N, Gardiner A, Sunter N (2021) Did the COVID-19 pandemic increase insomnia? Sleep 44:A263-A263. https://doi. org/10.1093/sleep/zsab072.671

24. Li Q, Guan X, Wu P et al (2020) Early transmission dynamics in Wuhan, China, of novel coronavirus-infected pneumonia. N Engl J Med 382(13):1199-1207. https://doi.org/10.1056/ NEJMoa2001316

25. Yuan S, Liao Z, Huang $\mathrm{H}$ et al (2020) Comparison of the indicators of psychological stress in the population of Hubei Province and non-endemic provinces in China during Two weeks during the coronavirus disease 2019 (COVID-19) outbreak in February 2020. Med Sci Monit 26:e923767. https://doi.org/10.12659/ MSM.923767

26. Chan KL, Chen M (2019) Effects of social media and mobile health apps on pregnancy care: meta-analysis. JMIR Mhealth Uhealth 7(1):e11836. https://doi.org/10.2196/11836 
27. De Rosa O, Cellini N, Conte F, Malloggi S, Giganti F, Ficca G (2021) Self-reported sleep features in the Italian population across the first and second wave of the COVID-19 pandemic. Sleep 44:A77-A78. https://doi.org/10.1093/sleep/zsab072.191

28. Lewis BA, Gjerdingen D, Schuver K, Avery M, Marcus BH (2018) The effect of sleep pattern changes on postpartum depressive symptoms. BMC Womens Health 18(1):12. https://doi.org/10. 1186/s12905-017-0496-6

29. Obeysekare JL, Cohen ZL, Coles ME et al (2020) Delayed sleep timing and circadian rhythms in pregnancy and transdiagnostic symptoms associated with postpartum depression. Transl Psychiatry 10(1):14. https://doi.org/10.1038/ s41398-020-0683-3

30. McMahon CA, Boivin J, Gibson FL et al (2011) Older first-time mothers and early postpartum depression: a prospective cohort study of women conceiving spontaneously or with assisted reproductive technologies. Fertil Steril 96(5):1218-1224. https://doi. org/10.1016/j.fertnstert.2011.08.037

31. Lin JL, Lin YH, Chueh KH (2014) Somatic symptoms, psychological distress and sleep disturbance among infertile women with intrauterine insemination treatment. J Clin Nurs 23(11-12):16771684. https://doi.org/10.1111/jocn.12306

32. Huang LH, Kuo CP, Lu YC, Lee MS, Lee SH (2019) Association of emotional distress and quality of sleep among women receiving in-vitro fertilization treatment. Taiwan J Obstet Gynecol 58(1):168-172. https://doi.org/10.1016/j.tjog.2018.11.031
33. Shayganfard M, Mahdavi F, Haghighi M, Sadeghi Bahmani D, Brand S(2020) Health anxiety predicts postponing or cancelling routine medical health care appointments among women in perinatal stage during the Covid-19 lockdown. Int J Environ Res Public Health 17(21). https://doi.org/10.3390/ijerph17218272

34. Shorey S, Chee CYI, Ng ED, Chan YH, Tam WWS, Chong YS (2018) Prevalence and incidence of postpartum depression among healthy mothers: a systematic review and meta-analysis. J Psychiatr Res 104:235-248. https://doi.org/10.1016/j.jpsychires.2018.08. 001

35. Ceulemans M, Hompes T, Foulon V (2020) Mental health status of pregnant and breastfeeding women during the COVID-19 pandemic: a call for action. Int J Gynaecol Obstet 151(1):146-147. https://doi.org/10.1002/ijgo.13295

36. Lillis TA, Hamilton NA, Pressman SD, Khou CS (2018) The association of daytime maternal napping and exercise with nighttime sleep in first-time mothers between 3 and 6 months postpartum. Behav Sleep Med 16(6):527-541. https://doi.org/10.1080/15402 002.2016.1239580

37. Cheng TS, Loy SL, Cheung YB et al (2017) Plasma vitamin D deficiency is associated with poor sleep quality and night-time eating at mid-pregnancy in Singapore. Nutrients 9(4). https://doi. org/10.3390/nu9040340

Publisher's note Springer Nature remains neutral with regard to jurisdictional claims in published maps and institutional affiliations. 